\title{
Generalizations of Bateman's transformation for general indefinite metrics
}

\author{
Katsunori SHImomURA*
}

\begin{abstract}
Bateman's transformation is associated with the Lorentzian metric and preserves solutions of the wave equation. We generalize Bateman's transformation for general indefinite semi-euclidean metrics. Then we show that the generalized transformation preserves solutions of the equation associated with given indefinite metric.
\end{abstract}

\section{Introduction}

Let $x, y, z, t$ be the coordinate of $\mathbb{R}^{4}$. In [1], Bateman introduced his transformation defined on $\{z-c t \neq 0\} \subset \mathbb{R}^{4}$, which transforms a function $w(x, y, z, t)$ to

$$
\frac{1}{z-c t} w(B(x, y, z, t)):=\frac{1}{z-c t} w\left(\frac{x}{z-c t}, \frac{y}{z-c t}, \frac{r^{2}-1}{2(z-c t)}, \frac{r^{2}+1}{2 c(z-c t)}\right),
$$

where $r^{2}=x^{2}+y^{2}+z^{2}-c^{2} t^{2}$. Bateman's transformation preserves the solutions of the wave equation

$$
\frac{\partial^{2} w}{\partial x^{2}}+\frac{\partial^{2} w}{\partial y^{2}}+\frac{\partial^{2} w}{\partial z^{2}}-\frac{1}{c^{2}} \frac{\partial^{2} w}{\partial t^{2}}=0
$$

Note that the above wave operator is associated with the indefinite metric

$$
\langle(x, y, z, t),(x, y, z, t)\rangle:=x^{2}+y^{2}+z^{2}-c^{2} t^{2} .
$$

In our previous paper [3], we determined the transformations which preserve the solution of the wave equation on $\mathbb{R}^{n}(n \geq 3)$. There we introduced a transformation which is a modification of (1.1). We called the transformation Bateman transformation, which played crucial roles in that paper. In [4], we gave some relations between the Lorentzian inversion and the Bateman transformation. The relations enable us to generalize the Bateman's transformation (1.1) naturally to general metrics.

Received 8 February, 2012; revised 25 March, 2013; revised 1 April 2013

2000 Mathematics Subject Classification. 31B99, 35K99, 35A30

Key Words and Phrases. semi-euclidean, wave equation, Bateman's transformation

* Partially supported by Grant-in-aid for Scientific Research (C) No.22540169, 23540220, Japan Society for the Promotion of Science.

*Ibaraki University, Mito, Ibaraki, 310-8512, Japan. (shimomur@mx.ibaraki.ac.jp) 
The key was the following relation. Let $K(x, y, z, t)=\left(\frac{x}{r^{2}}, \frac{y}{r^{2}}, \frac{z}{r^{2}}, \frac{t}{r^{2}}\right)$ be the Lorentzian inversion, and put $S(x, y, z, t)=\left(x, y, z-\frac{1}{2}, t+\frac{1}{2 c}\right)$ and $T(x, y, z, t)=$ $\left(x, y, z+\frac{1}{2}, t+\frac{1}{2 c}\right)$. Then $B, K, S$, and $T$ satisfy

$$
B=S K T K S \text {. }
$$

The purpose of this note is to generalize the Bateman's transformation to general indefinite semi-euclidean metrics by making use of a relation corresponds to (1.2) and to show that the generalized transformation preserve the solutions of the equation associated with the given indefinite metric.

\section{2. $U_{\gamma}$-morphism}

Let $\mathbb{R}^{n}$ be the $n$-dimensional Euclidean space $(n \geq 2)$, and denote its point by $x=\left(x_{1}, \ldots, x_{n}\right)$. Let $\gamma=\left(\gamma_{i j}\right)$ be a non-degenerate symmetric $(n, n)$-matrix of signature $(p, q)$ where $p+q=n$ and $1 \leq p, q \leq n-1$. We define an indefinite scalar product on $\mathbb{R}^{n}$ by

$$
\langle u, v\rangle=\langle u, v\rangle_{\gamma}=\sum_{i, j=1}^{n} \gamma_{i j} u_{i} v_{j}
$$

We consider the second order partial differential operator associated with $\gamma$ on $\mathbb{R}^{n}$

$$
U_{\gamma} w=\sum_{i, j=1}^{n} \gamma^{i j} \frac{\partial^{2} w}{\partial x_{i} \partial x_{j}}
$$

where $\left(\gamma^{i j}\right)$ is the inverse matrix of $\left(\gamma_{i j}\right)$.

Definition 2.1. $\quad$ Let $D \subset \mathbb{R}^{n}$ be an open set, $f: D \rightarrow \mathbb{R}^{n}$ a $C^{2}$-mapping, and $\varphi$ a non-vanishing $C^{2}$-function on $D$. A pair $(f, \varphi)$ is called a $U_{\gamma}$-morphism, if $f$ and $\varphi$ satisfy the following conditions:

(1) $f^{\prime}(x)$ is non-degenerate for all $x \in D$.

(2) For every solution $w$ of the equation $U_{\gamma} w=0$ on $f(D), \varphi \cdot(w \circ f)$ satisfies the equation $U_{\gamma}(\varphi \cdot(w \circ f))=0$ on $D$.

Here, $f^{\prime}(x): \mathbb{R}^{n} \rightarrow \mathbb{R}^{n}$ is the first derivative of $f$ at $x \in D$ defined by $f^{\prime}(x) v=$ $\lim _{h \rightarrow 0} \frac{f(x+h v)-f(x)}{h}$.

By definition, it is easy to see that if $\left(f, \varphi_{f}\right)$ and $\left(g, \varphi_{g}\right)$ are $U_{\gamma}$-morphisms such that the image of $f$ is contained in the domain of $g$, then $\left(g \circ f,\left(\varphi_{g} \circ f\right) \cdot \varphi_{f}\right)$ is also a $U_{\gamma}$-morphism. We call this transformation $\left(g \circ f,\left(\varphi_{g} \circ f\right) \cdot \varphi_{f}\right)$ the composition of $\left(f, \varphi_{f}\right)$ and $\left(g, \varphi_{g}\right)$. $\left.\gamma^{-1}\right\}$

We list some examples of $U_{\gamma}$-morphism. Let $\mathrm{O}_{\gamma}(n)=\left\{R \in \mathrm{GL}(n, \mathbb{R}) ; R \gamma^{-1 t} R=\right.$

Example 2.1 (similarity). Let $\alpha, C \in \mathbb{R}, \alpha>0, C \neq 0, R \in \mathrm{O}_{\gamma}(n), a \in \mathbb{R}^{n}$,

$$
f(x)=\alpha R x+a, \quad \varphi(x)=C .
$$

Then the pair $(f, \varphi)$ is a $U_{\gamma}$-morphism on $\mathbb{R}^{n}$. We call this transformation similarity. 
Example 2.2 (inversion). Let $D=\{x ;\langle x, x\rangle \neq 0\}$ and let

$$
K(x)=\frac{1}{\langle x, x\rangle} x, \quad \varphi_{K}(x)=|\langle x, x\rangle|^{\frac{2-n}{2}} .
$$

Then the pair $\left(K, \varphi_{K}\right)$ is a $U_{\gamma}$-morphism defined on $D$. We call this transformation inversion.

In fact, $K$ satisfies $K^{2}=I$ and this implies that $K^{\prime}(x)$ is non degenerate on $D$. Next, by the chain rule,

$$
\begin{array}{r}
U_{\gamma}(\varphi \cdot(w \circ K))=\sum_{i, j=1}^{n} \gamma^{i j}\left(\frac{\partial^{2} \varphi}{\partial x_{i} \partial x_{j}} \cdot(w \circ K)+2 \frac{\partial \varphi}{\partial x_{i}} \frac{\partial}{\partial x_{j}}(w \circ K)+\varphi \cdot \frac{\partial^{2}}{\partial x_{i} \partial x_{j}}(w \circ K)\right) \\
=\left(U_{\gamma} \varphi\right) \cdot(w \circ K)+\sum_{k=1}^{n}\left(2 \sum_{i, j=1}^{n} \gamma^{i j} \frac{\partial \varphi}{\partial x_{i}} \frac{\partial K_{k}}{\partial x_{j}}+\varphi \sum_{i, j=1}^{n} \gamma^{i j} \frac{\partial^{2} K_{k}}{\partial x_{i} \partial x_{j}}\right) \cdot\left(\frac{\partial w}{\partial y_{k}} \circ K\right) \\
+\varphi \sum_{k, l=1}^{n}\left(\sum_{i, j=1}^{n} \gamma^{i j} \frac{\partial K_{k}}{\partial x_{j}} \frac{\partial K_{l}}{\partial x_{i}}\right) \cdot\left(\frac{\partial^{2} w}{\partial y_{l} \partial y_{k}} \circ K\right)
\end{array}
$$

holds. Since

$$
\begin{gathered}
\sum_{i, j=1}^{n} \gamma^{i j} \frac{\partial^{2} \varphi}{\partial x_{i} \partial x_{j}}=0, \quad \sum_{i, j=1}^{n} \gamma^{i j} \frac{\partial \varphi}{\partial x_{i}} \frac{\partial K_{k}}{\partial x_{j}}=-(2-n) \frac{x_{k}}{\langle x, x\rangle^{2}} \varphi \\
\sum_{i, j=1}^{n} \gamma^{i j} \frac{\partial^{2} K_{k}}{\partial x_{i} \partial x_{j}}=2(2-n) \frac{x_{k}}{\langle x, x\rangle^{2}}, \quad \sum_{i, j=1}^{n} \gamma^{i j} \frac{\partial K_{l}}{\partial x_{i}} \frac{\partial K_{k}}{\partial x_{j}}=\frac{\gamma^{l k}}{\langle x, x\rangle^{2}},
\end{gathered}
$$

we have

$$
U_{\gamma}(\varphi \cdot(w \circ K))=\varphi \sum_{k, l=1}^{n}\left(\frac{\partial^{2} w}{\partial y_{l} \partial y_{k}} \circ K\right) \frac{\gamma^{k l}}{\langle x, x\rangle^{2}}=\frac{\varphi}{\langle x, x\rangle^{2}}\left(\left(U_{\gamma} w\right) \circ K\right),
$$

which shows that $(K, \varphi)$ is a $U_{\gamma}$-morphism.

\section{Generalization of Bateman's transformation}

First we prepare the involution associated with the metric. Let

$$
\gamma=|\gamma| J, \quad\left(|\gamma|=\sqrt{{ }^{t} \gamma \gamma}, J \in \mathrm{O}(n)\right)
$$

be the polar decomposition of $\gamma$. Since $\gamma$ is real symmetric, $|\gamma|=\sqrt{\gamma^{2}},|\gamma|$ is also real symmetric, and hence $\gamma|\gamma|=|\gamma| \gamma$ holds. Therefore the matrices $\gamma, \gamma^{-1},|\gamma|,|\gamma|^{-1}, J, J^{-1}$ are mutually commutative. Hence

$$
J^{2}=I, \quad J \in \mathrm{O}_{\gamma}(n)
$$

holds because $J \gamma^{-1 t} J=\gamma^{-1} J^{2}=\gamma^{-1}$. Since $\gamma J=\gamma^{2}|\gamma|^{-1}=|\gamma|$ is positive definite,

$$
\langle x, J x\rangle_{\gamma}=\langle x, x\rangle_{|\gamma|}>0 \quad(x \neq 0) .
$$


Let $u \in \mathbb{R}^{n}$ satisfy $u \neq 0$ and $\langle u, u\rangle=0$. We define a conjugate of $u$ by

$$
u^{*}=\frac{J u}{\langle u, J u\rangle} \text {. }
$$

Then

$$
\left(u^{*}\right)^{*}=u, \quad\left\langle u^{*}, u^{*}\right\rangle=0
$$

because $J^{2}=I$. Since $\left\langle u, u^{*}\right\rangle=1, u$ and $u^{*}$ are linearly independent. Note that

$$
\begin{gathered}
(\alpha u)^{*}=\frac{1}{\alpha} u^{*} \quad(\alpha>0), \\
\left\langle\alpha u+\beta u^{*}, \alpha u+\beta u^{*}\right\rangle=2 \alpha \beta \quad(\alpha, \beta \in \mathbb{R})
\end{gathered}
$$

For $a \in \mathbb{R}^{n}$, we define the translation $T_{a}$ by $T_{a}(x)=x-a$. Clearly $T_{a}(A x)=A T_{A^{-1} a}(x)$ holds for any $A \in \mathrm{GL}(n, \mathbb{R})$.

Now we generalize Bateman's mapping and transformation. Let $u \in \mathbb{R}^{n}$ satisfy $u \neq 0$ and $\langle u, u\rangle=0$. We define the $(\gamma, u)$-Bateman mapping $B_{u}$ on $\{x ;\langle x, u\rangle \neq 0\}$ by

$$
B_{u}(x)=T_{u^{*}} K T_{-\frac{1}{2} u} K T_{u^{*}}(x) .
$$

Next we put

$$
\varphi_{B_{u}}(x)=|\langle x, u\rangle|^{\frac{2-n}{2}} .
$$

We call the pair $\left(B_{u}, \varphi_{B_{u}}\right)(\gamma, u)$-Bateman transformation.

This $(\gamma, u)$-Bateman transformation is a generalization of the original Bateman's transformation (see Remark 1 below).

In our previous papers [3] and [4], we called the mapping $B=B_{u} J$ Bateman mapping, where $\gamma=\operatorname{diag}(-1,1, \ldots, 1)$ and $u=(1,1,0, \ldots, 0)$.

\section{Results}

Theorem 4.1. Let $u \in \mathbb{R}^{n}$ with $u \neq 0$ and $\langle u, u\rangle=0$. Then $B_{u}$ and $\varphi_{B_{u}}$ satisfy the following.

(1)

$$
B_{u}(x)=\frac{1}{\langle x, u\rangle}\left(\frac{\langle x, x\rangle}{2} u-u^{*}+Q_{u} x\right)
$$

where $Q_{u} x=x-\left\langle x, u^{*}\right\rangle u-\langle x, u\rangle u^{*}$ is a linear mapping.

(2) $B_{u}^{-1}=B_{-u}$.

(3) $\left(B_{u}, \varphi_{B_{u}}\right)$ is a $U_{\gamma}$-morphism.

Proof. We first prove (1). Since $\langle u, u\rangle=0,\left\langle u^{*}, u^{*}\right\rangle=0$, and $\left\langle u, u^{*}\right\rangle=1$,

$$
\left(K T_{-\frac{1}{2} u} K\right)(y)=\frac{y+\frac{1}{2}\langle y, y\rangle u}{1+\langle y, u\rangle+\frac{1}{4}\langle y, y\rangle\langle u, u\rangle}=\frac{y+\frac{1}{2}\langle y, y\rangle u}{1+\langle y, u\rangle}=\frac{y+\frac{1}{2}\langle y, y\rangle u}{\left\langle u^{*}, u\right\rangle+\langle y, u\rangle} .
$$


Putting $x=y+u^{*}=T_{u^{*}}^{-1}(y)$, the right hand side equals to

$$
\begin{aligned}
\frac{x-u^{*}+\frac{1}{2}\left\langle x-u^{*}, x-u^{*}\right\rangle u}{\langle x, u\rangle} & =\frac{1}{\langle x, u\rangle}\left(x-u^{*}+\frac{\langle x, x\rangle}{2} u-\left\langle x, u^{*}\right\rangle u\right) \\
& =\frac{1}{\langle x, u\rangle}\left(\frac{\langle x, x\rangle}{2} u-u^{*}+Q_{u} x\right)+u^{*} .
\end{aligned}
$$

Therefore $B_{u}(x)=T_{u^{*}} K T_{-\frac{1}{2} u} K T_{u^{*}}(x)=\frac{1}{\langle x, u\rangle}\left(\frac{\langle x, x\rangle}{2} u-u^{*}+Q_{u} x\right)$ holds.

Next, since $(-u)^{*}=-u^{*}$ and $K^{-1}=K$,

$$
B_{u}^{-1}=T_{-u^{*}} K^{-1} T_{\frac{1}{2} u} K^{-1} T_{-u^{*}}=T_{(-u)^{*}} K T_{-\frac{1}{2}(-u)} K T_{(-u)^{*}}=B_{-u} .
$$

Finally, to show (3), it suffices to show that $\left(B_{u}, \varphi_{B_{u}}\right)$ is the composition of $U_{\gamma^{-}}$ morphisms $\left(T_{u^{*}}, 1\right),\left(K, \varphi_{K}\right),\left(T_{-\frac{u}{2}}, 1\right),\left(K, \varphi_{K}\right)$, and $\left(T_{u^{*}}, 1\right)$. In fact, since

$$
\begin{aligned}
\left\langle T_{-\frac{1}{2} u} K(y), T_{-\frac{1}{2} u} K(y)\right\rangle\langle y, y\rangle & =\left\langle\frac{y}{\langle y, y\rangle}+\frac{u}{2}, \frac{y}{\langle y, y\rangle}+\frac{u}{2}\right\rangle\langle y, y\rangle \\
& =1+\langle u, y\rangle=\left\langle u, u^{*}+y\right\rangle,
\end{aligned}
$$

by substituting $y=T_{u^{*}}(x)=x-u^{*}$ into above, we have

$$
\left\langle T_{-\frac{1}{2} u} K T_{u^{*}}(x), T_{-\frac{1}{2} u} K T_{u^{*}}(x)\right\rangle\left\langle T_{u^{*}}(x), T_{u^{*}}(x)\right\rangle=\langle u, x\rangle .
$$

Then

$$
\begin{aligned}
\varphi_{T_{u^{*}} K T_{-\frac{1}{2} u} K T_{u^{*}}(x)} & =1 \cdot \varphi_{K}\left(T_{-\frac{1}{2} u} K T_{u^{*}}(x)\right) \cdot 1 \cdot \varphi_{K}\left(T_{u^{*}}(x)\right) \cdot 1 \\
& =\varphi_{K}\left(T_{-\frac{1}{2} u} K T_{u^{*}}(x)\right) \varphi_{K}\left(T_{u^{*}}(x)\right) \\
& =|\langle u, x\rangle|^{\frac{2-n}{2}}=\varphi_{B_{u}}(x) .
\end{aligned}
$$

Therefore, $\left(B_{u}, \varphi_{B_{u}}\right)$ is a $U_{\gamma}$-morphism.

Remark 1. The above $\left(B_{u}, \varphi_{B_{u}}\right)$ is actually a generalization of the original Bateman's transformation. If $n=4, \gamma=\operatorname{diag}\left(1,1,1,-c^{2}\right)$ and $u=\left(0,0,1, \frac{1}{c}\right)$, then $J=\operatorname{diag}(1,1,1,-1), u^{*}=\left(0,0, \frac{1}{2},-\frac{1}{2 c}\right),\langle x, u\rangle=x_{3}-c x_{4}$, and $Q_{u} x=\left(x_{1}, x_{2}, 0,0\right)$. By (4.1), $B_{u}(x)=\frac{1}{x_{3}-c x_{4}}\left(x_{1}, x_{2}, \frac{\langle x, x\rangle-1}{2}, \frac{\langle x, x\rangle+1}{2 c}\right)$ on $D=\left\{x \in \mathbb{R}^{4} ; x_{3}-c x_{4}>0\right\}$ and $(\gamma, u)$-Bateman transformation is

$$
\frac{1}{x_{3}-c x_{4}} w\left(\frac{x_{1}}{x_{3}-c x_{4}}, \frac{x_{2}}{x_{3}-c x_{4}}, \frac{\langle x, x\rangle-1}{2\left(x_{3}-c x_{4}\right)}, \frac{\langle x, x\rangle+1}{2 c\left(x_{3}-c x_{4}\right)}\right),
$$

which is equal to Bateman's transformation on $D$.

Proposition 4.1. Let $u$ satisfy $u \neq 0$ and $\langle u, u\rangle=0$ and let $\alpha \in \mathbb{R} \backslash\{0\}$. Then the linear map $Q_{u}$ has the following properties;

$$
\begin{array}{cl}
Q_{u} u=Q_{u} u^{*}=0, \quad Q_{u}^{2}=Q_{u}, & \left\langle Q_{u} x, u\right\rangle=\left\langle Q_{u} x, u^{*}\right\rangle=0 \\
Q_{\alpha u}=Q_{u}=Q_{u^{*}}=Q_{J u}, & \left\langle Q_{u} x, y\right\rangle=\left\langle x, Q_{u} y\right\rangle .
\end{array}
$$


Proof. First, $Q_{u} u=Q_{u} u^{*}=0$ and $\left\langle Q_{u} x, u\right\rangle=\left\langle Q_{u} x, u^{*}\right\rangle=0$ follows from $\langle u, u\rangle=$ $\left\langle u^{*}, u^{*}\right\rangle=0$ and $\left\langle u, u^{*}\right\rangle=1$. Then $Q_{u} u=Q_{u} u^{*}=0$ implies $Q_{u}^{2}=Q_{u}$. Since $\left(u^{*}\right)^{*}=u$ and $(\alpha u)^{*}=\alpha^{-1} u^{*}, Q_{\alpha u}=Q_{u}=Q_{u^{*}}=Q_{J u}$ holds. Finally, $\left\langle Q_{u} x, y\right\rangle=\left\langle x, Q_{u} y\right\rangle$ follows by interchanging $x$ and $y$ in $\left\langle Q_{u} x, y\right\rangle=\langle x, y\rangle-\left\langle x, u^{*}\right\rangle\langle u, y\rangle-\langle x, u\rangle\left\langle u^{*}, y\right\rangle$.

Remark 2. The above proposition suggests that every $x \in \mathbb{R}^{n}$ can be written as $x=\left\langle x, u^{*}\right\rangle u+\langle x, u\rangle u^{*}+Q_{u} x$ and the vectors $u, u^{*}, Q_{u} x$ are mutually orthogonal with respect to $\gamma$. Conversely, if $x \in \mathbb{R}^{n}$ can be written as $x=\alpha u+\beta u^{*}+z$ with $\alpha, \beta \in \mathbb{R}$ and $\langle u, z\rangle=\left\langle u^{*}, z\right\rangle=0$, then $z=Q_{u} x$. Therefore we can regard $Q_{u}$ as a " $\gamma$-orthogonal projection to the complement of the subspace spanned by $u$ and its adjoint $u^{* ”}$.

By definition, $(\gamma, u)$-Bateman transformation is a composition of inversion and translations. Conversely, inversion is a composition of $(\gamma, u)$-Bateman transformation and similarities. We introduce a linear map $S_{v} x:=x-\frac{2\langle x, v\rangle}{\langle v, v\rangle} v$ for $v$ with $\langle v, v\rangle \neq 0$.

Proposition 4.2. $\quad$ For $v$ with $\langle v, v\rangle \neq 0$ and $\alpha \in \mathbb{R} \backslash\{0\}, S_{v}$ satisfies

$$
S_{\alpha v}=S_{v}, \quad S_{v} \in \mathrm{O}_{\gamma}(n), \quad S_{v}^{2}=I, \quad\left\langle\frac{x+S_{v} x}{2}, v\right\rangle=0 .
$$

Proof. $\quad S_{\alpha v}=x-\frac{2\langle x, \alpha v\rangle}{\langle\alpha v, \alpha v\rangle} \alpha v=x-\frac{2\langle x, v\rangle}{\langle v, v\rangle} v=S_{v}$ by definition. $S_{v} \in \mathrm{O}_{\gamma}(n)$ because

$$
\left\langle S_{v} x, S_{v} x\right\rangle=\langle x, x\rangle-4 \frac{\langle x, v\rangle}{\langle v, v\rangle}\langle x, v\rangle+4 \frac{\langle x, v\rangle^{2}}{\langle v, v\rangle^{2}}\langle v, v\rangle=\langle x, x\rangle .
$$

Since $\left\langle S_{v} x, v\right\rangle=\langle x, v\rangle-2 \frac{\langle x, v\rangle}{\langle v, v\rangle}\langle v, v\rangle=-\langle x, v\rangle,\left\langle\frac{x+S_{v} x}{2}, v\right\rangle=0$ and $S_{v}^{2} x=S_{v} x-2 \frac{\left\langle S_{v} x, v\right\rangle}{\langle v, v\rangle} v=S_{v} x+2 \frac{\langle x, v\rangle}{\langle v, v\rangle} v=x$.

Remark 3. The above proposition suggests that $S_{v}$ can be regarded as a "reflection with respect to the hyperplane which is $\gamma$-orthogonal to $v "$.

Theorem 4.2. Let $u \in \mathbb{R}^{n}$ satisfy $u \neq 0$ and $\langle u, u\rangle=0$. Put $v=u+J u$. Then

$$
K=\frac{\langle u, J u\rangle}{2} S_{v} B_{u} B_{J u}=\frac{\langle u, J u\rangle}{2} S_{v} B_{u} J B_{u} J
$$

holds. This shows that the inversion is a composition of Bateman transformations and similarities.

Proof. First we shall show $J B_{u} J=B_{J u}$. Since $J \in \mathrm{O}_{\gamma}(n)$ and $J^{2}=I$,

$$
\begin{aligned}
J B_{u} J(x) & =\frac{1}{\langle J x, u\rangle}\left(\frac{\langle J x, J x\rangle}{2} J u-J u^{*}+J\left(J x-\left\langle J x, u^{*}\right\rangle u-\langle J x, u\rangle u^{*}\right)\right) \\
& =\frac{1}{\langle x, J u\rangle}\left(\frac{\langle x, x\rangle}{2} J u-(J u)^{*}+x-\left\langle x,(J u)^{*}\right\rangle J u-\langle x, J u\rangle(J u)^{*}\right) \\
& =B_{J u} .
\end{aligned}
$$


Therefore, it suffices to show the first equality in (4.2). Since $\left\langle(J u)^{*}, u\right\rangle=0, Q_{J u}=Q_{u}$ and $\left\langle Q_{u} x, u\right\rangle=0$,

$$
\left\langle B_{J u}(x), u\right\rangle=\frac{1}{\langle x, J u\rangle} \frac{\langle x, x\rangle}{2}\langle J u, u\rangle .
$$

Since $\left\langle Q_{u} x, Q_{u} x\right\rangle=\left\langle Q_{u} x, x\right\rangle$ and $\left\langle Q_{u} x-x, x\right\rangle=-2\langle x, u\rangle\left\langle u^{*}, x\right\rangle$,

$$
\left\langle B_{J u}(x), B_{J u}(x)\right\rangle=\frac{1}{\langle x, J u\rangle^{2}}\left(\left\langle Q_{u} x, Q_{u} x\right\rangle-\langle x, x\rangle\right)=-\frac{2\langle x, u\rangle}{\langle x, J u\rangle\langle u, J u\rangle} .
$$

$Q_{u}^{2}=Q_{u}$ and $Q_{u} u=Q_{u} u^{*}=0$ imply

$$
Q_{u} B_{J u}(x)=\frac{1}{\langle x, J u\rangle} Q_{u} x .
$$

Note that $\langle v, v\rangle=\langle u+J u, u+J u\rangle=2\langle u, J u\rangle>0$. Therefore

$$
\begin{aligned}
B_{u} B_{J u}(x) & =\frac{1}{\left\langle B_{J u}(x), u\right\rangle}\left(\frac{\left\langle B_{J u}(x), B_{J u}(x)\right\rangle}{2} u-u^{*}+Q_{u} B_{J u}(x)\right) \\
& =\frac{2\langle x, J u\rangle}{\langle x, x\rangle\langle J u, u\rangle}\left(-\frac{\langle x, u\rangle u}{\langle x, J u\rangle\langle u, J u\rangle}-\frac{J u}{\langle u, J u\rangle}+\frac{Q_{u} x}{\langle x, J u\rangle}\right) \\
& =\frac{2}{\langle x, x\rangle\langle J u, u\rangle}\left(-\frac{\langle x, u\rangle u}{\langle u, J u\rangle}-\frac{\langle x, J u\rangle J u}{\langle u, J u\rangle}+x-\frac{\langle x, J u\rangle u}{\langle u, J u\rangle}-\frac{\langle x, u\rangle J u}{\langle u, J u\rangle}\right) \\
& =\frac{2}{\langle x, x\rangle\langle J u, u\rangle}\left(x-\frac{\langle x, u+J u\rangle(u+J u)}{\langle v, v\rangle / 2}\right) \\
& =\frac{2}{\langle J u, u\rangle}\left(\frac{x}{\langle x, x\rangle}-\frac{2\left\langle\frac{x}{\langle x, x\rangle}, v\right\rangle v}{\langle v, v\rangle}\right)=\frac{2}{\langle J u, u\rangle} S_{v} K(x) .
\end{aligned}
$$

Since $S_{v}^{2}=I, K(x)=\frac{\langle J u, u\rangle}{2} S_{v} B_{u} B_{J u}(x)$.

\section{Acknowledgments}

The authors would like to express our gratitude to the referee for his valuable comments.

\section{References}

[1] H. Bateman, The conformal transformations of a space of four dimensions and their applications to geometrical optics, Proc. London Math. Soc., 7 (1909) $70-$ 89 .

[2] B. O'Neill, Semi-Riemannian geometry, Academic Press, 1983.

[3] K. Shimomura, Liouville type theorem associate with the wave equation, Math. J. Ibaraki Univ., 43 (2011), $51-64$.

[4] K. Shimomura, A relation between the Lorentzian inversion and the Bateman transformation, Math. J. Ibaraki Univ., 44 (2012), 1 - 5. 